\title{
Avaliação da performance muscular de idosas não sedentárias antes e após aplicação de um programa de exercícios de equilíbrio
}

\section{Eveluetion of msalar performanceinnd sedentary ddaly beforeand atter theapdication of an exerciseprogramfor balane}

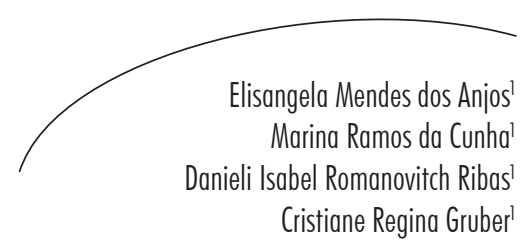

Resumo

Introdução: Uma das alterações do processo natural de senescência é a diminuição da massa e força muscular, denominada de sarcopenia. Caracterizada por um processo lento e progressivo no idoso, afetará a funcionalidade e a qualidade de vida, aumentando a morbidade, dependência, hospitalizações recorrentes, riscos de quedas e fraturas. Objetivo: Avaliar os efeitos dos exercícios de equilíbrio na força e na flexibilidade de idosos. Métodos: 13 idosas com idade de 60 a 83 anos (73 anos \pm DP 5,15), voluntárias e praticantes de atividade física, foram avaliadas antes e após aplicação de um programa de equilíbrio, realizado por 12 semanas consecutivas, uma vez na semana, em relação à força e flexibilidade de membros inferiores. A força foi avaliada por meio do teste de sentar e levantar, e a flexibilidade, do teste de sentar e alcançar. Os resultados foram analisados por meio do teste paramétrico ANOVA ( $\mathrm{p} \geq 0,05)$ Resultados: Não ocorreram ganhos significativos ( $\mathrm{p} \geq 0,05)$ em relação à força e à flexibilidade, porém ocorreu manutenção dos seus valores. Conclusão: 12 semanas de aplicação de exercícios de equilíbrio, realizados uma vez na semana, são suficientes para manutenção da força e flexibilidade de idosas não sedentárias.

\section{Abstract}

Introduction: One of the natural processes of senescence is the decrease in muscle strength and mass, called sarcopenia, a slow and progressive process that in the elderly will affect the functionality and quality of life by increasing morbity, dependence, recurrent hospitalizations, risk of falls and fractures. Objective: To evaluate the effects of balance exercises on the strength and flexibility in elderly. Methods: 13 volunteered elderly women aged 60 to 83 years ( 73 years \pm 5.15 ), engaged in physical activity were evaluated for strength and flexibility of the lower limbs before and after the application of a balance program, for 12 consecutive weeks, once a week. Strength was evaluated using the test sitting and standing and the flexibility through the sit and reach test. The results were analyzed by ANOVA ( $\mathrm{p} \geq 0.05)$. Results: There was no significant gains $(\mathrm{p} \geq 0,05)$ in the strength and flexibility, but there was a maintaining in the values. Conclusion: 12 weeks of application of balance exercises, performed once a week are enough to maintain strength and flexibility of non-sedentary elderly.

Curso de Fisioterapia, Faculdades Integradas do Brasil. Curitiba, PR, Brasil

Palavras-chave: Exercícios de equilíbrio. Idoso. Mulheres. Força muscular e flexibilidade.

Key words: Balance exercises. Elderly. Women. Muscle strength and flexibility. 


\section{INTRODUÇÃO}

De acordo com Gonçalves, Gurjão \& Gobbi, ${ }^{1}$ a força e a flexibilidade muscular são essenciais no que diz respeito à realização de atividades de vida diária. Com o passar da idade, ocorre uma diminuição desses fatores, levando o idoso a apresentar uma diminuição da sua qualidade de vida, aumentando o risco de quedas e a perda da mobilidade articular. ${ }^{2-4}$ À medida que se envelhece, ocorre declínio das reservas fisiológicas, como a diminuição da função imunológica, do colágeno e da reserva cardíaca. Ocorrem modificações da síntese e do metabolismo proteico, admitindo-se como regra que $1 \%$ da função se perde a cada ano após os 30 anos de idade. ${ }^{2,5,6}$

Uma das alterações do processo natural de senescência é a diminuição da massa e força muscular, denominada de sarcopenia, um processo lento e progressivo que no idoso afetará a funcionalidade e a qualidade de vida, aumentando a morbidade, a dependência, as hospitalizações recorrentes, os riscos de quedas e as fraturas. ${ }^{5,7}$ Com o passar dos anos, o músculo esquelético perde massa e força, devido à diminuição de sua área de secção transversa e perda de unidades motoras. ${ }^{3,8-10}$ Entre os 25 e 80 anos, ocorre redução de 40 a 50\% de massa muscular, ${ }^{9,10}$ maior em membros inferiores, quando comparada aos membros superiores. ${ }^{6}$

Essas alterações senescentes são comuns, mas seu aparecimento pode ser acelerado pelo sedentarismo, em que o sedentarismo irá aumentar a predisposição ao desenvolvimento de doenças cardiovasculares e outras condições crônico-degenerativas. ${ }^{5,11}$

A atividade física é provavelmente o melhor investimento na saúde para as pessoas em processo de envelhecimento. ${ }^{10,12}$ Além de contribuir para a melhora da flexibilidade e da força, aumenta a perspectiva de vida, minimiza os efeitos degenerativos provocados pelo envelhecimento, permitindo ao idoso manter uma melhor qualidade de vida ativa. ${ }^{13-16} \mathrm{~A}$ prática regular de atividade física tem evidenciado capacidade para atenuar grande parte das alterações fisiológicas causadas pelo envelhecimento. Estudos mostram que a prática de atividade física está relacionada com a síntese mineral óssea, prevenindo assim o risco de fraturas. ${ }^{17,18}$

Estudos têm descrito que o exercício aeróbio, exercícios resistidos e alongamentos realizados isoladamente ou em conjunto promovem benefícios imediatos e em longo prazo, como redução do risco de quedas, melhora da capacidade cardiorrespiratória, flexibilidade e força muscular. No entanto, a literatura é escassa quanto aos benefícios de exercícios de equilíbrio na população idosa para ganho de força e flexibilidade.

De acordo com Costa et al., ${ }^{19}$ a prática de atividades de equilíbrio, além de aumentar a autoconfiança em pessoas idosas, proporciona melhora nas capacidades funcionais, melhorando seu desempenho nas tarefas do cotidiano que demandam equilíbrio, minimizando principalmente os riscos de quedas.

De acordo com a literatura, exercícios com peso, aeróbicos e atividades na água promovem a manutenção do alongamento muscular, mas a prática de exercícios de equilíbrio irá promover ajustes do centro de gravidade, causando contrações isométricas seguidas de relaxamento, o que pode levar a manutenção e/ou ganho do comprimento e força muscular. Desta forma, o presente estudo teve como objetivo avaliar os efeitos dos exercícios de equilíbrio, realizados uma vez na semana, na força e na flexibilidade de idosos, antes e após 12 semanas de treinamento.

\section{MATERIAIS E MÉTODOS}

Este estudo longitudinal, aprovado pelo Comitê de Ética e Pesquisa da Unibrasil sob o parecer 037/2009, foi realizado com 13 idosas com idade de 60 a 83 anos (73 anos $\pm 5,15)$, voluntárias, não sedentárias, na cidade de Curitiba, participantes de um grupo da terceira idade e que realizavam caminhadas duas vezes na semana. 
Foram incluídas no estudo idosas que praticassem atividade física, voluntárias com mais de 60 anos. Foram excluídas do estudo idosas que apresentassem qualquer condição que impossibilitasse a realização da avaliação e dos exercícios propostos, tais como hipertensão descontrolada, uso de órteses, sequelas motoras de AVC, amputações e uso de cadeiras de rodas, e os que não apresentassem o Termo de Consentimento Livre e Esclarecido assinado.

Para a classificação do grupo estudado em não sedentário, utilizou-se o Questionário Internacional de Atividade Física (IPAQ), versão curta, o qual permitiu classificar os indivíduos em três categorias de nível de atividade física (baixo, moderado e alto), de acordo com as diretrizes do Colégio Americano do Esporte (ACMS). A amostra do estudo apresentou os níveis recomendados de atividade física (moderado e alto), sendo, portanto, classificada em não sedentária.
As idosas selecionadas foram submetidas a avaliação da força e da flexibilidade muscular de membros inferiores. Como forma de avaliação da força muscular, utilizou-se o teste sentar e levantar; ${ }^{20}$ e para testar a flexibilidade, foi utilizado o teste sentar e alcançar. ${ }^{21}$

Para a realização do teste de sentar e levantar, a idosa foi posicionada em uma cadeira, com os braços cruzados sobre o peito. $\mathrm{O}$ teste consistiu em levantar e sentar da cadeira o maior número de vezes durante 30 segundos. Foram realizadas duas tentativas, sendo a primeira para familiarização do exercício. A segunda tentativa foi realizada após a idosa referir estar apta para sua execução.

Para o teste de flexibilidade, foi necessário utilizar uma fita adesiva nas medidas $50,8 \mathrm{~cm}$, fixada ao solo, e uma fita métrica que também foi fixada ao solo perpendicularmente, sendo suas marcas de $63,5 \mathrm{~cm}$ diretamente colocadas sobre a fita adesiva. Foram feitas duas marcas equidistantes $15 \mathrm{~cm}$ do centro da fita métrica (figura 1).

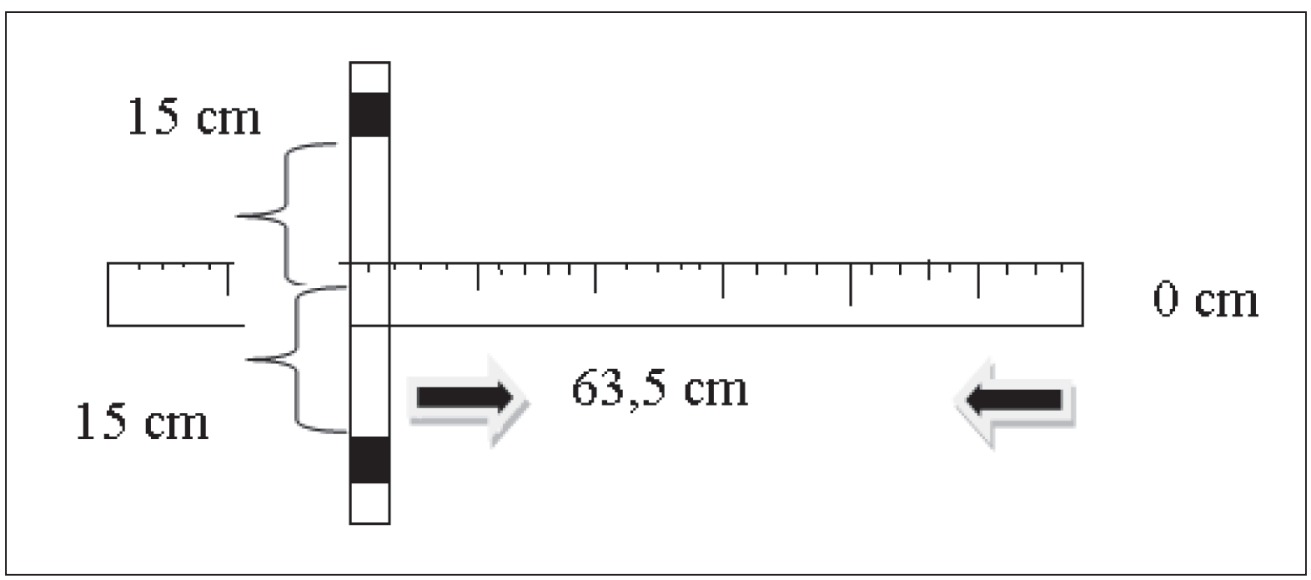

Figura 1 - Marcação do teste de flexibilidade.

Para a realização do teste, cada idosa tinha que estar descalça; sentava-se com os membros inferiores estendidos e pés afastados $30,4 \mathrm{~cm}$ entre si, os artelhos apontando para cima e os calcanhares centrados nas marcas feitas na fita adesiva. O zero da fita métrica apontava para a participante. Com as mãos uma sobre a outra, vagarosamente, deslizava as mãos sobre a fita métrica tão distante quanto conseguisse, assim permanecendo na posição final no mínimo dois segundos. O avaliador auxiliou, segurando o joelho da participante, evitando assim que o mesmo se flexionasse. Foram concedidas duas tentativas de prática, seguidas de duas tentativas de teste, sendo o resultado final o melhor obtido nas tentativas. ${ }^{21}$ 
As idosas foram submetidas à avaliação da força e flexibilidade muscular antes, seis e 12 semanas após o início do programa de exercícios. Nos dias das avaliações, foi solicitado as idosas que suspendessem a realização de qualquer tipo de atividade física. Após a avaliação inicial, as idosas foram submetidas a um programa de exercícios para equilíbrio. Estes exercícios foram dispostos em circuito e aplicados uma vez na semana por um período de 12 semanas, com duração de 50 minutos cada sessão, totalizando 12 sessões (quadro 1).

O programa foi dividido em três etapas. Exercícios de aquecimento (5 minutos) como alongamentos, atividades lúdicas com bexigas, bolas e músicas. Exercícios de equilíbrio dispostos em circuito; que eram realizados três vezes. Após três semanas, o circuito era mudado, mas eram usados os mesmos materiais e o mesmo objetivo; na primeira etapa eram realizados sete exercícios e, na segunda etapa, seis exercícios; e exercícios de relaxamento (5 minutos) músicas calmas, automassagem, massagem em dupla. No início de cada sessão, foi realizada a verificação dos dados vitais (pressão arterial e frequência cardíaca) como forma de controle.

Os dados coletados foram inseridos em planilhas e os resultados foram submetidos à análise por meio do teste paramétrico ANOVA considerando o intervalo de $95 \%$ de significância $(\mathrm{p} \leq 0,05)$.

Quadro 1 - Sequência dos exercícios de equilíbrio aplicados no estudo. Curitiba, PR, 2009.

\begin{tabular}{|c|c|}
\hline \multicolumn{2}{|c|}{ Exercícios } \\
\hline Primeira Sequência & Segunda Sequência \\
\hline $\begin{array}{l}\text { 1) O indivíduo sentado deverá levantar e contornar } \\
\text { seis cones. }\end{array}$ & $\begin{array}{l}\text { 1) Percorrer uma distância de dois metros, entrar } \\
\text { em quatro bambolês dispostos no chão, pegá-los e } \\
\text { caminhar em direção a um tatame disposto ao chão. }\end{array}$ \\
\hline $\begin{array}{l}\text { 2) Ultrapassar seis obstáculos de diferentes alturas } \\
\text { e larguras dispostos em linha reta. } \\
\text { 3) Com uma bola nas mãos, deverá caminhar em } \\
\text { superfícies diferenciadas (areia, espuma, pedra e } \\
\text { terra); ao final do caminho deverá arremessar a } \\
\text { bola dentro de um cesto. }\end{array}$ & $\begin{array}{l}\text { 2) Sentar e levantar de uma cadeira, contornar dois } \\
\text { obstáculos (cone), caminhar sobre duas almofadas } \\
\text { (espuma, arreia), subir e descer dois degraus, } \\
\text { contornar dois cones e caminhar sobre duas } \\
\text { almofadas (terra, pedra) sentar em um cadeira e } \\
\text { levantar/sentar cinco vezes. }\end{array}$ \\
\hline $\begin{array}{l}\text { 4) Contornar quatro cones, subir e descer dois } \\
\text { degraus e em seguida chutar uma bola. }\end{array}$ & $\begin{array}{l}\text { 3) Segurando um bastão, ultrapassar seis } \\
\text { obstáculos dispostos em zigue-zague. }\end{array}$ \\
\hline $\begin{array}{l}\text { 5) Andar sobre uma linha reta, pegar uma bola e } \\
\text { arremessar na "boca do palhaço". }\end{array}$ & $\begin{array}{l}\text { 4) Contornar três obstáculos, pular amarelinha, } \\
\text { abaixar para pegar uma bola disposta no chão e } \\
\text { arremessá-la em um cesto. }\end{array}$ \\
\hline $\begin{array}{l}\text { um pé na frente do outro. } \\
\text { 7) Levantar de uma cadeira, passar por cima de um } \\
\text { colchonete, andar por dentro de quatro bambolês }\end{array}$ & $\begin{array}{l}\text { 5) Segurando uma bola, caminhar de lado sobre } \\
\text { um colchonete, ao final do percurso chutar a bola } \\
\text { em direção ao gol. }\end{array}$ \\
\hline $\begin{array}{l}\text { com os pés alternados, por um colchonete, por } \\
\text { mais quatro bambolês, pisar em mais um bambolê } \\
\text { e andar sobre um tatame pequeno com um pé a } \\
\text { frente do outro. }\end{array}$ & $\begin{array}{l}\text { 6) Contornar três obstáculos; ao final do percurso, } \\
\text { pegar três bolas de tênis dispostas no chão e } \\
\text { arremessá-las na "boca do palhaço". }\end{array}$ \\
\hline
\end{tabular}


RESULTADOS

Os resultados obtidos em relação a força e flexibilidade antes (avaliação 1) e após (avaliação
2) aplicação dos exercícios de equilíbrio dispostos em circuito serão apresentados a seguir. $\mathrm{Na}$ tabela 1, estão dispostos os valores referentes à caracterização da amostra do estudo.

Tabela 1 - Caracterização da amostra ( $\mathrm{n}=13)$ em relação ao número de participantes, sexo e as médias de idade, peso, estatura e IMC. Curitiba, PR, 2009.

\begin{tabular}{cc}
\hline Variável & Amostra \\
\hline Número de participante $(\mathrm{n})$ & 13 \\
Sexo $(\mathrm{n})$ feminino & 13 \\
Idade (anos) média + DP & $73.69 \pm 5.15$ \\
Peso $(\mathrm{Kg})$, media + DP & $69.07 \pm 15.11$ \\
Estatura $(\mathrm{cm})$ média + DP & $1.52 \pm 0.063$ \\
IMC $(\mathrm{Kg} / \mathrm{cm})$ média + DP & $28.2 \pm 5.56$ \\
\hline
\end{tabular}

Os resultados obtidos em relação à força muscular de membros inferiores antes (avaliação
1) e após (avaliação 2) aplicação dos exercícios de equilíbrio podem ser visualizados na figura 2.

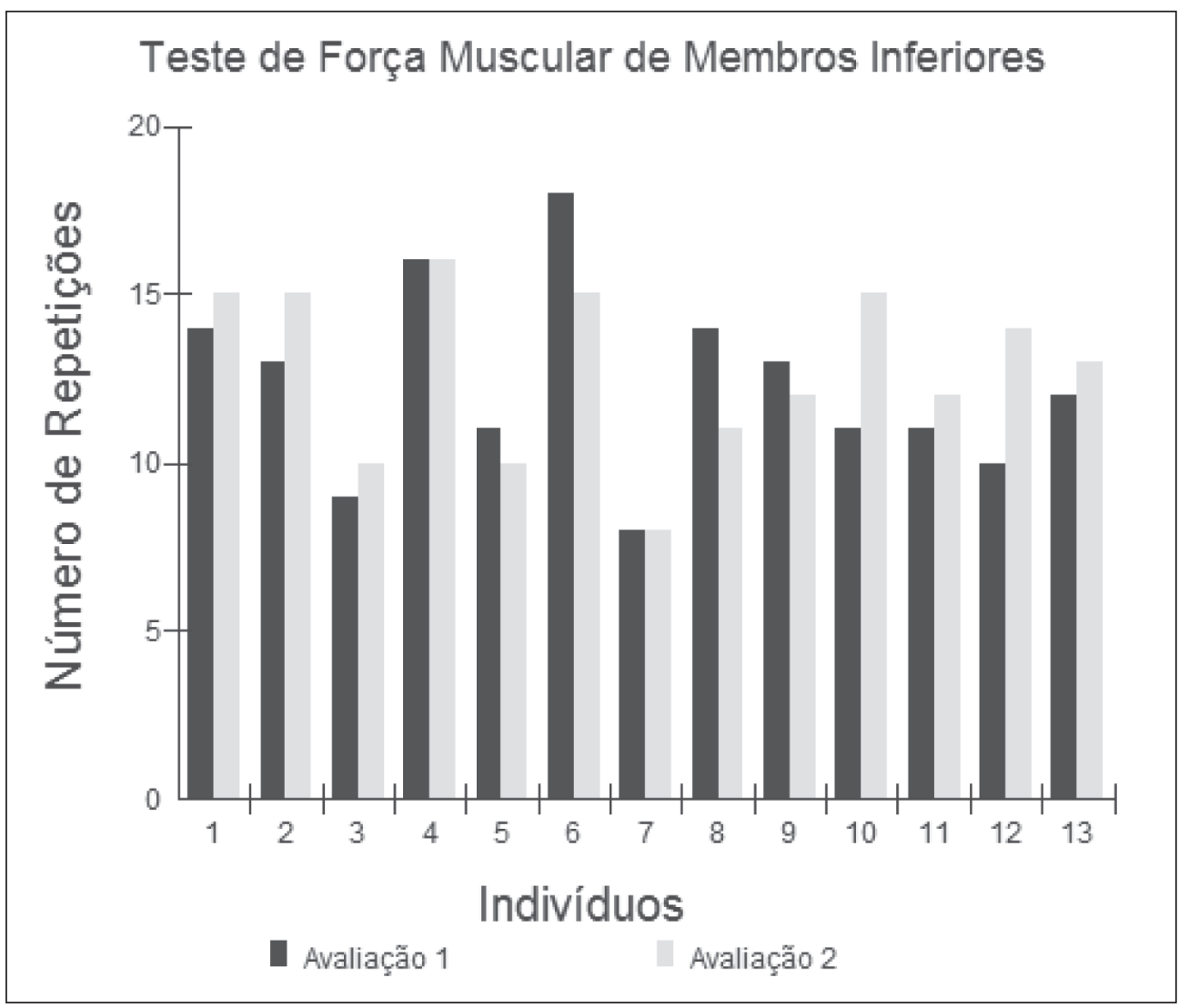

Figura 2 - Valores referentes à força muscular de membros inferiores obtidos pré (avaliação 1) e pós (avaliação 2) aplicação do programa de exercícios de equilíbrio. 
Ao analisar os valores obtidos para a força muscular, é possível verificar que o valor médio encontrado antes da aplicação dos exercícios foi igual a 12,3 ( $\pm 2,78$ ) repetições e o valor após aplicação foi de $12,76( \pm 2,48)$ repetições, não ocorrendo diferença significativa entre os valores ( $p \geq 0,05)$.
Apesar de a maioria dos indivíduos apresentarem aumento em seus valores após aplicação do programa de exercícios de equilíbrio este aumento também não foi significativo $(p \geq 0,05)$. Os valores referentes à flexibilidade de membros inferiores podem ser observados na figura 3 .

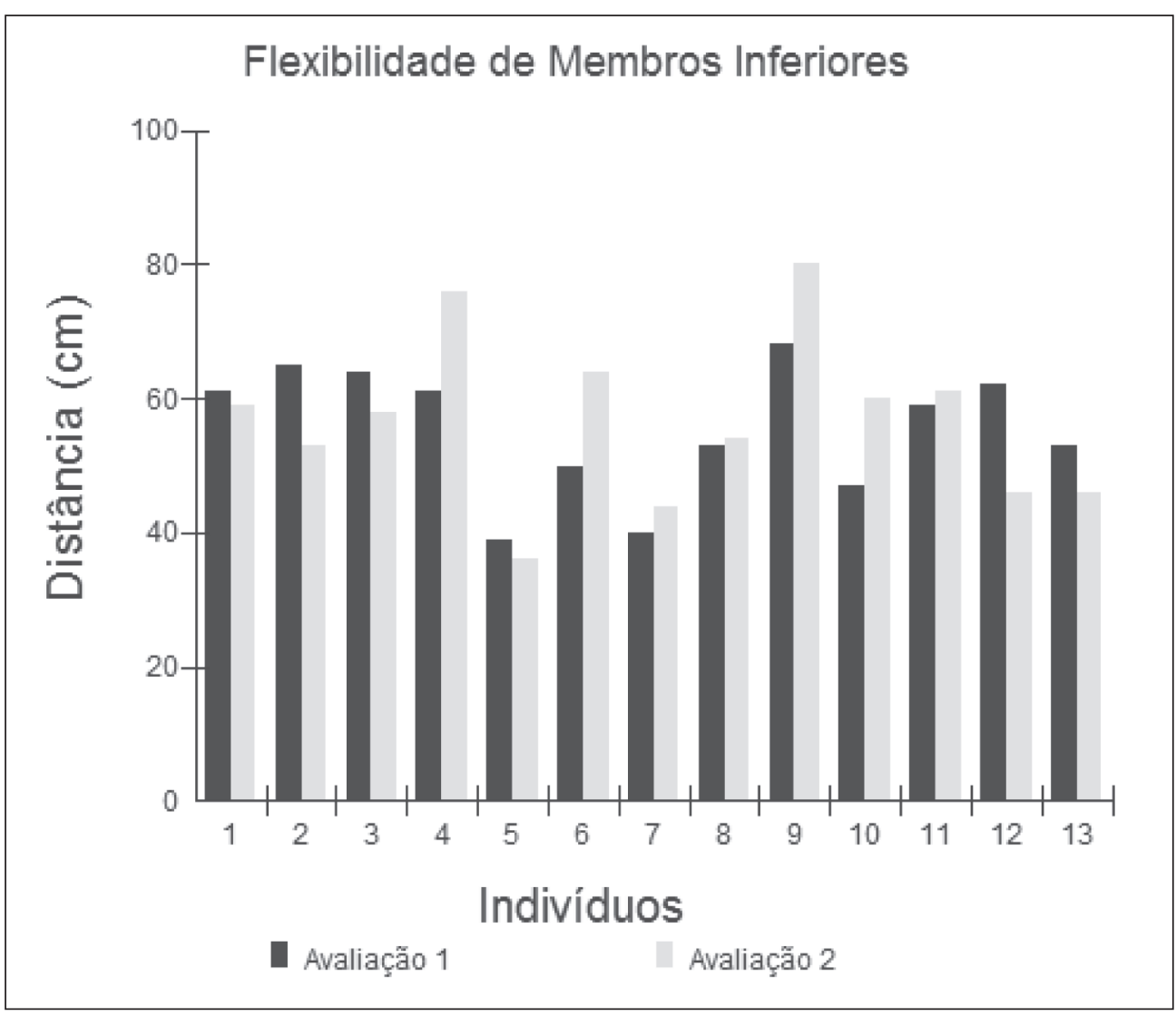

Figura 3 - Valores referentes à flexibilidade muscular de membros inferiores obtidos antes (avaliação 1) e após (avaliação 2) aplicação do programa de exercícios de equilíbrio

Ao comparar os valores médios encontrados antes e após aplicação do programa de exercícios de equilíbrio, percebe-se que estes foram de $55,53 \mathrm{~cm}( \pm 9,4)$ antes e $56,69 \mathrm{~cm}( \pm 12,39)$ após os exercícios. Apesar de ocorrer aumento médio de $1,16 \mathrm{~cm}$ para a flexibilidade, este não foi significativo ( $\mathrm{p} \geq 0,05)$. $\mathrm{E}$, ao analisar os resultados individualmente, nota-se que as idosas 4,6 e 9 apresentaram aumento de 15, 14 e $12 \mathrm{~cm}$, respectivamente, destacando-se das demais.

\section{DISCUSSÃO}

O presente estudo avaliou a flexibilidade e a força muscular de membros inferiores de idosas não sedentárias, antes e após aplicação de 12 semanas de exercícios de equilíbrio dispostos em circuito e realizados uma vez na semana. Os resultados obtidos demonstraram que, após aplicação dos exercícios propostos, não houve melhora significativa na força e na flexibilidade da 
amostra do estudo, mas ocorreu manutenção dos valores. Mota et al. ${ }^{22}$ e Benedetti \& Benedetti ${ }^{23}$ relatam que os idosos praticantes de atividade física mostraram melhor desempenho quando comparados com idosos que não praticam nenhuma atividade. ${ }^{23}$

Esses resultados são semelhantes aos encontrados por Gonçalves, Gurjão \& Gobbi ${ }^{1}$ e Rebelatto et al. ${ }^{10}$ Os primeiros autores constataram em seu estudo que exercícios com peso não promovem ganho da flexibilidade, mas impedem a sua perda. E o segundo autor verificou que um programa de exercícios de força, potência, atividades de coordenação, agilidade, flexibilidade, exercícios respiratórios e de relaxamento, realizado três vezes por semana durante 58 semanas, foram suficientes apenas para manter os processos naturais da senescência. Cabe salientar que o programa de equilíbrio do presente estudo foi realizado uma vez na semana e mostrou manutenção das variáveis flexibilidade e força, fato de extrema importância, pois a manutenção destas variáveis, de acordo com Rebelatto et al., ${ }^{10}$ leva à melhora da independência funcional dos idosos.

Os resultados obtidos neste estudo podem ser explicados pelo fato de o programa de exercícios de equilíbrio proposto não estar associado a exercícios de alongamento muscular. Os alongamentos musculares utilizados no aquecimento apresentavam valores inferiores de manutenção e número de repetições preconizadas pela literatura para proporcionar ganho de flexibilidade. ${ }^{24,25}$

Estudos anteriores mostram que exercícios de alongamento muscular, quando realizados sozinhos ou associados a outros tipos de exercícios, promovem ganhos de flexibilidade em idosos. ${ }^{8,26-29}$ Silva et al., ${ }^{5}$ em seu estudo comparativo, relatam que as atividades de alongamento, exercícios aeróbicos, localizados, coreografias, relaxamento e atividades lúdicas, realizadas em grupo, duas vezes na semana e com 60 minutos de duração, também são significativas para melhorar a flexibilidade muscular.
Os resultados encontrados para a força muscular de membros inferiores são semelhantes aos encontrados por Candeloro \& Caromano, ${ }^{30}$ que verificaram que um programa de hidroterapia não foi capaz de promover alterações significativas na força muscular de idosas. No entanto, diferem no que se diz respeito à flexibilidade: o mesmo programa, quando analisado em relação aos ganhos de flexibilidade, se mostrou eficiente.

Estudo realizado com agachamento em plataforma vibratória também mostrou não ser eficiente para melhorar a força muscular. ${ }^{20}$ A literatura mostra que exercícios com peso, associados ou não a outros tipos de exercícios realizados uma ou duas vezes na semana são capazes de promover ganho de força muscular em idosos. ${ }^{8,31,32}$ Isto pode explicar por que não ocorreu melhora da força muscular com a aplicação do programa de equilíbrio, pois este não apresentava associação com exercícios de peso. Apesar de os idosos realizarem agachamentos, estes não eram sistematizados em séries e repetições, nem realizados contra resistência.

Quando comparados os resultados entre os grupos de indivíduos considerados idosos e velhos, percebeu-se que o grupo classificado como idoso apresentou melhores valores para a força muscular, não ocorrendo diferença para a flexibilidade.

Pereira et al. ${ }^{33}$ relatam que, mesmo quando os programas de atividade física não apresentam especificidade do treinamento de força, eles ainda podem proporcionar uma manutenção da mesma. Rogatto \& Gobbi, ${ }^{34}$ ao compararem a força muscular de mulheres jovens e idosas praticantes de atividade física, concluíram que mesmo realizando atividade física, os níveis de força muscular tendem a diminuir com o passar da idade, mas a prática da mesma tende a manter a força muscular das idosas. Raso ${ }^{35}$ cita, em seu estudo, que um programa de exercícios com pesos de baixa intensidade pode produzir efeitos similares ao de programas de alta intensidade. 
Outro fator que pode ter influenciado os resultados do estudo foi o tamanho da amostra, um número maior de indivíduos avaliados poderia proporcionar maior fidedignidade dos resultados. Também devem ser considerados fatores como motivação e habituação na realização dos testes e dos exercícios propostos. No entanto, os cuidados realizados durante a execução do estudo foram suficientes para garantir sua validade interna.

\section{CONCLUSÃO}

A aplicação de exercícios de equilíbrio uma vez na semana proporcionou manutenção da força muscular e flexibilidade de idosas não sedentárias. Essa manutenção pode ser explicada pelos próprios benefícios dos exercícios de equilíbrio, pois durante sua execução ocorrem contrações isométricas seguidas de relaxamento. Mesmo os exercícios não sendo específicos, eles foram capazes de manter as variáveis avaliadas e promover diminuição dos efeitos do envelhecimento.

Novos estudos devem ser realizados utilizando exercícios de equilíbrio estático e dinâmico, com frequência maior de realização na semana e com uma amostra maior, com o intuito de avaliar os benefícios desses exercícios nessa população.

\section{REFERÊNCIAS}

1. Gonçalves R, Gurjão ALD, Gobbi S. Efeitos de oito semanas do treinamento de força na flexibilidade de idosos. Revista Brasileira de Cineantropometria e Desempenho Humano 2007 jun; 2 (9): 145-153.

2. Holland GJ, et al. Flexibility and physical functions of older adults: A Review. J Aging Phys Act. 2002; 10(2).169-206.

3. ACSM (American College of Sports Medicine). The recommended quantity and quality of exercise for developing and maintaining cardiorespiratory and muscular fitness in healthy adults. Med Sci Sports Exerc. 1998; 30(6):975-991.

4. Vale RGS, Aragão JCB, Dantas EHM. A Flexibilidade na autonomia funcional de idosas independentes. Fit. Perf. J. 2003 jan/fev; 2 (1): 23-29.

5. Silva TAA, Frisoli AJ, Pinheiro MM, Szejnfeld VL. Sarcopenia associada ao envelhecimento: aspectos etiológicos e opções terapêuticas. Revista Brasileira de Reumatologia 2006 nov/dez; 6 (46): 391-95.

6. Carvalho J, Soares JMC. Envelhecimento e força muscular: breve revisão. Revista Brasileira de Ciência do Desporto 2004; 3 (4): 79-93.

7. Doherty TJ. Physiology of aging invited review: aging and sarcopenia. The American Physiological Society 2003 nov; (95):1717-1727.

8. Vale RGS, Barreto ACG, Novaes JS, Dantas EHM. Efeitos do treinamento resistido na força máxima, na flexibilidade e na autonomia funcional de mulheres idosas. Revista Brasileira Cineantropometria e Desempenho Humano 2006; 4 (8): 52-8.
9. Vale RGS et al,. Efeitos do treinamento de força na flexibilidade de mulheres idosas.Fit.Perf.J. 2004; 5 (3): 266-271.

10. Rebelatto JR, Calvo JI, Orejuela JR, Portillo JC. Influência de um programa de atividade física de longa duração sobre a força muscular manual e a flexibilidade corporal de mulheres idosas. Rev Bras de Fisiot 2006; 1 (10):127-132.

11. Guimarães LHCT, et al. Comparação da propensão de quedas entre idosos que praticam atividade física e idosos sedentários. Rev de Neuroc 2004 abr/jun; 2 (12):68-72.

12. Jong $\mathrm{N}$, et al. Functional biochenical and nutrient indices in frail elderly people are partly affected by dietary supplements but not by exercise. The Journal of Nutrition 1999 jul.129:2028-2036.

13. Rocha AC, et al. Análise comparativa da força muscular entre idosas praticantes de musculação, ginástica localizada e institucionalizada. Journal Fit. Perf.J. 2009 jan/fev; 8 (1):16-20.

14. Mello R, et al. Tai Chi e autonomia: comparação do grau de flexibilidade e autonomia em idosas praticantes de Tai Chi e sedentárias.Fit. Perf. J. 2004 jul/ago; 3(4):194-200.

15. Cortes GG, Silva VF. Força e autonomia: manutenção da força muscular e autonomia em mulheres idosas, conquistadas em trabalho prévio de adaptação neural. Fit.Perf.J. 2005; 4(2):1007-1017.

16. Paula RH, Vale RGS, Dantas EHM. Eletromiografia: relação entre o nível de autonomia funcional de 
adultos idosos com grau de fadiga muscular aguda periférica verificado pela eletromiografia. Fit. Perf. J. 2006; 5(2):95-100.

17. Kerr D, et al. Resistence training over 2 years increases bone mass in calcium-replete postmenopausal woman. J Bone Miner Res. 2001; 1 (16): 175-181.

18. Nelson ME, et al. Effects of high-intensity strength training on multiple risk factors for osteoporotic fractures. Jama 1994 dec; 24 (272):1909-1914.

19. Costa J N A. Exercicios multisensoriais no equilíbrio e na prevenção de quedas em idosos. Rev Dig Buenos Aires 2009 ago;135(14).

20. Silva RG, et al. Efeito do treinamento vibratório na força muscular e em testes funcionais em idosos fisicamente ativos Rev.Bras.Cineantropom desempenho Hum 2009 abr; 11 (2): 166-173.

21. Benedetti TR, et al. Valores normativos de aptidão funcional em mulheres de 70 a 79 anos. Rev Bras. Cineantropom Desempenho Hum 2007; 1(9): 28-33.

22. Mota J, et al. Atividades físicas e qualidade de vida associadas à saúde em idosos participantes e não participantes em programas regulares de atividades físicas. Ver. Bras. de Educ. Fis 2006 jul; 20 (3): 219-225.

23. Benedetti TRB, Benedetti AL. Musculação na Terceira Idade. Rev de Educ. Fis/UEM 1996; 7(1): 35-40.

24. Feland JB. The effect of duration of stretching of the hamstring muscle group for increasing range of motion in people aged 65 years or older. Physical Therapy 2001 may; 5 (81): 1110-1117.

25. Bandy WD, Irion JM, Briggler M. The effect of time and frequency of static stretching on flexibility of the hamstring muscles. Physical Therapy 1997 oct; 10 (77): 1090-1096.

26. Lanuez FV, Jacob WF. Efeitos de dois programas de exercícios físicos nos determinantes de aptidão motora em idosos sedentários. Einstein 2008; 1 (6):76-81.
27. Gama, ZAS, et al. Influencia do intervalo de tempo entre as sessões de alongamento no ganho de flexibilidade dos isquiotibiais. Rev. Bras. de Med Esporte 2009 mar/abr; 2 (15): 110-14.

28. Gama, ZAS et al. Influência da freqüência de alongamento utilizando facilitação neuromuscular proprioceptiva na flexibilidade dos músculos isquiotibiais. Rev.Bras.Med Esporte 2007 jan/fev; 1 (13): 33-8.

29. Fatouros LG, et al. Resistance training and detraining effects on flexibility performance in the elderly are intensity-dependent. Journal Strength Training and Elderly Flexibility 2006 aug; 20 (3):634-642.

30. Candeloro JM, Caromano FA. Efeito de um programa de hidroterapia na flexibilidade e na força muscular de idosas. Rev. Bras Fisio 2007 jul/ago; 4 (11): 303-09.

31. Donoghue JD, Werner W, Douris PC. Comparison of once-weekly and twice-weekly strength training in older adults. J Br Sports Med. 2007; (41): 19-22.

32. Aveiro MC, et al. Influence of a physical training programo n muscle strength, balance and gait velocity among women with osteoporosis. Rev. Bras. Fisio 2006 out/dez; 4 (10): 441-48.

33. Pereira FD, et al. Comparação da força funcional de membros inferiores e superiores entre idosas fisicamente ativas e sedentárias. Rev. Bras. Geriatri e Gerontol. 2009;12(3): 417-427.

34. Rogatto GP, Gobbi S. Efeitos da atividade física regular sobre parâmetros antropométricos e funcionais de mulheres jovens e idosas. Revista Brasileira de Cineantropometria e Desempenho Humano 2001; 3(1): 63-69.

35. Raso V. Exercícios com peso para pessoas idosas: a experiência do Celafiscs. Rev. Bras. Ciên. e Mov. 2000 mar;8(2):43-51. 